\title{
Efficacy of low-dose radiotherapy in painful gonarthritis: experiences from a retrospective East German bicenter study
}

Stephanie Keller ${ }^{1 \dagger}$, Klaus Müller ${ }^{2 *}$, Rolf-Dieter Kortmann², Ulrich Wolf², Guido Hildebrandt ${ }^{3}$, André Liebmann², Oliver Micke ${ }^{4}$, Gert Flemming ${ }^{5}$ and Dieter Baaske ${ }^{6}$

\begin{abstract}
Purpose: To evaluate the efficacy of low-dose radiotherapy in painful gonarthritis.

Methods: We assessed the medical records of 1037 patients with painful gonarthritis who had undergone low-dose radiotherapy between 1981 and 2008. The subjective patient perception of the response to irradiation as graded immediately or up to two months after the completion of a radiotherapy series was evaluated and correlated with age, gender, radiological grading and the duration of symptoms before radiotherapy. Moreover, we performed a mail survey to obtain additional long-term follow-up information and received one hundred and six evaluable questionnaires.

Results: We assessed 1659 series of radiotherapy in 1037 patients. In 79.3\% of the cases the patients experienced a slight, marked or complete pain relief immediately or up to two months after the completion of radiotherapy. Gender, age and the duration of pain before radiotherapy did not have a significant influence on the response to irradiation. In contrast, severe signs of osteoarthritis were associated with more effective pain relief. In more than $50 \%$ of the patients who reported a positive response to irradiation a sustained period of symptomatic improvement was observed.

Conclusions: Our results confirm that low-dose radiotherapy is an effective treatment for painful osteoarthritis of the knee. In contrast to an earlier retrospective study, severe signs of osteoarthritis constituted a positive prognostic factor for the response to irradiation. A randomized trial is urgently required to compare radiotherapy with other treatment modalities.
\end{abstract}

Keywords: Osteoarthritis, Knee, Radiotherapy, Irradiation, X-ray, Gonarthritis, Gonarthrosis, Osteoarthrosis

\section{Background}

Painful osteoarthritis of the knee is one of the world's most common degenerative joint disorders [1,2]. Its analgetic treatment with low-dose ionizing radiation has a long tradition in Germany [3-5]. Nevertheless, the acceptance of this method, especially abroad, has to be considered low. Accordingly, radiotherapy still has not been included as a therapeutic option in the European League against Rheumatism (EULAR) guidelines [6] and

\footnotetext{
* Correspondence: Klaus.Mueller@medizin.uni-leipzig.de

${ }^{\dagger}$ Equal contributors

${ }^{2}$ Department of Radiotherapy, University Hospital of Leipzig, Stephan-Str. 9a, Leipzig 04103, Germany

Full list of author information is available at the end of the article
}

usually represents the "last resort" before surgery. Until recently the pain-relieving effect of radiotherapy in painful gonarthritis was mainly demonstrated by earlier studies, which were highly susceptible to criticism due to methodological weaknesses, outdated radiation techniques and partly low numbers of patients. In 2010 the German Cooperative Group on Radiotherapy for Benign Diseases (GCG-BD) resumed the issue again releasing the results of a large pattern of care study on the role of radiotherapy in painful and refractory gonarthritis which had been conducted in 42 German radiotherapy institutions from 2006 to 2008. High response and low toxicity could be demonstrated in a very large number $(n=5069)$ of cases [5]. However, one may object that

\section{Ciomed Central}


there are methodological problems that are inherent to this kind of survey. The quality of data can be verified only with great difficulty and the information may reflect rough estimates or personal opinions. The purpose of this retrospective bicenter study was to provide evidence that radiotherapy is effective in the treatment of painful gonarthritis and thus can be a reasonable alternative to other therapeutic options. Furthermore we investigated the influence of possible prognostic factors on the pain relieving effect of radiotherapy. We finally performed a systematic literature search and discussed our results in the light of recent releases.

\section{Methods}

The clinical data of 1037 patients who had undergone radiotherapy for painful gonarthritis in the hospitals of Chemnitz and Aue between 1981 and 2008 were evaluated retrospectively. The diagnosis was based on medical history, orthopaedic examination, which was performed by the referring physician and/or the radiotherapist and a conventional X-ray examination. The classification of the radiological severity of gonarthritis in "normal", "minimal", "moderate" and "severe" was made by entries in the patient files using the Kellgren-Lawrence score [7]. Most of the patients were treated using orthovoltage units with a radiation of $150,175,180$ or $200 \mathrm{kV}, 20 \mathrm{~mA}$ and copper filters of 0.5 or $1.0 \mathrm{~mm}$ thickness. The focus-skin distance was 40 or $50 \mathrm{~cm}$. Some patients were treated on a linear accelerator with 6 or $9 \mathrm{MeV}$ photons or on a radiotherapeutic unit with a Cs-137 radiation source in the 1980s. The joints were irradiated from medial and lateral and in some exceptional cases from anterior and posterior with field sizes ranging between $8 \times 10$ and $15 \times 20 \mathrm{~cm}^{2}$. Radiotherapy was performed once a week in 611/1659 series (36.8\%), twice a week in $1045 / 1659$ series $(63.0 \%)$ or daily in $3 / 1659$ series $(0.2 \%)$. The single (total) doses ranged between 0.5 and 1.5 Gy (0.5 and 10 Gy) for a series (Table 1). As a matter of common practice two different radiotherapy techniques were applied. In Aue two opposed irradiation fields were used and the reference point of the dose was placed on the skin surface. In Chemnitz the knee joints were irradiated using a lateral and a medial (or a ventral and a dorsal) field in alternation and the reference point of the dose was placed in the center of the joint. The response to treatment was recorded, as it was subjectively graded by the patient immediately or up to two months after completion of treatment. Improvement of pain was categorized based on the classification published by von Pannewitz in 1933 [8] (painless, markedly improved, improved, stable, worse). In order to complete missing follow-up information questionnaires were mailed to 248 patients who had been treated in Chemnitz between 1996 and 2008. One hundred and six evaluable questionaires (42.7\%) were finally returned. The questionnaires addressed
Table 1 Summary of the fractionation schemes $\&$ dose

\section{prescriptions}

\begin{tabular}{lll}
\hline Item & $\mathbf{N}=$ & $\%$ \\
\hline Fractions per week & 1045 & 63.0 \\
1 & 611 & 36.8 \\
2 & 3 & 0.2 \\
5 & & \\
Single dose (Gy) & 248 & 14.9 \\
0,5 & 1389 & 83.7 \\
1 & 22 & 1.3 \\
Other & & \\
Total dose (Gy) & 899 & 54.2 \\
4 & 122 & 7.4 \\
6 & 638 & 38.5 \\
Other & 1659 & 100 \\
Total &
\end{tabular}

In 1037 patients with painful osteoarthritis of the knee who underwent radiotherapy (1659 series) between 1981 and 2008.

the pain relief after treatment in a four-stage classification, the ability to move in a three-level classification and the period during which a possible improvement had been observed.

\section{Statistical analysis}

Potential prognostic factors as age, gender, radiological grading and the duration of pain before radiotherapy were correlated with the response to irradiation using the chi square test. Statistical analysis was performed using MS Excel 2007 and SPSS 15.0.

\section{Literature search}

We performed Pubmed and Web of Science search with predefined search terms. The search was limited to English- and German-language articles published since 1980. English key words were: osteoarthritis, knee, radiotherapy, irradiation, $\mathrm{x}$-ray, gonarthritis, gonarthrosis, osteoarthrosis. German key words were: Osteoarthrose (itis), Arthrose (itis), Knie, Röntgenreizbestrahlung, Bestrahlung, Radiotherapie, Gonarthrose (itis). We identified 109 articles and abstracts by the search terms. After title and abstract review a preclinical [9] and four clinical articles [5,10-12] about radiotherapy in painful gonarthritis were eligible. In addition, we performed hand search following the references from selected articles.

\section{Ethical principles}

This retrospective study is in compliance with the Declaration of Helsinki - Ethical Principles for Medical Research Involving Human Subjects - and its amendments. 


\section{Results}

\section{Patient characteristics}

All in all 1659 series of radiotherapy in 1037 patients were assessed (Table 2). Three hundred sixteen patients were male $(30.5 \%)$ and 721 female (69.5\%). The age at the beginning of the first radiotherapy series ranged from 23 to 93 years. Patients younger than 40 years accounted for only a small proportion of the whole cohort $(n=15 / 1037,1.5 \%)$. Usually this group included patients who were suffering from post-traumatic osteoarthritis and had severe pain. The group of fourty to sixty-year-old was represented by a total of 304 patients (29.3\%). The group of over-sixty-year-old constituted the majority of patients $(\mathrm{n}=718 / 1037,69.2 \%) .662$ patients $(63.8 \%)$ underwent only a single series. 237 patients (22.9\%) received two, 84 patients $(8.1 \%)$ three and 54 patients (5.2\%) more than three series. On average, each patient was irradiated with 1.6 series. $11.4 \%$ of the patients who presented more than once were treated on both knees. Women were significantly more likely to undergo various series of radiotherapy on the same knee than men. $42.6 \%$ of the women were treated with two or more irradiation series, for men, the proportion was $37.6 \%$. In the majority of cases pain symptoms had occurred more than three years prior to the first irradiation ( $n=439 / 1037,42.4 \%)$. Twenty-one percent of the patients each suffered pain for less than a year $(n=213 / 1037)$ and for one to three years $(n=215 / 1037)$ respectively. Information about the radiological severity of arthritis before the first irradiation was available in 471 joints, which were irradiated with 654 series. 228 of these 654 series were effected to knees with moderate osteoarthritis. The knees with radiologically proven

Table 2 Patient characteristics

\begin{tabular}{lll}
\hline Item & $\mathbf{N}=$ & $\%$ \\
\hline Gender & $316 / 1037$ & 30.5 \\
Male & $721 / 1037$ & 69.5 \\
Female & & \\
Age group & $319 / 1037$ & 30.8 \\
$\leq 60$ years & $718 / 1037$ & 69.2 \\
$>60$ years & & \\
Severity & $119 / 651$ & 18.3 \\
Minimal & $228 / 651$ & 35.0 \\
Moderate & $304 / 651$ & 46.7 \\
Severe & & \\
Duration of pain & $213 / 867$ & 24.6 \\
$<1$ year & $215 / 867$ & 50.6 \\
1 - 3 years & $439 / 867$ & \\
$>3$ years &
\end{tabular}

Clinical data of 1037 patients with painful osteoarthritis of the knee who underwent radiotherapy between 1981 and 2008 . severe osteoarthritis received 308/654 series. In 119/654 series the joints showed minimal signs of osteoarthritis. $3 / 654$ series were effected to joints that were normal on imaging. $4.2 \%$ of the treated knees depicted axial deformity. The varus deformity was described nearly five times more often than the valgus deformity $(82.5 \%$ versus $17.5 \%)$. A traumatic pre-damage of the knee cold be elicited in $12.5 \%$ of the irradiated knees. Overall, $63.9 \%$ of the patients reported the application of alternative pain-killing treatments before the first radiotherapy series. The intra-articular administration of corticosteroids was first in line.

\section{Response to treatment as recorded immediately or up to two months after radiotherapy}

Reliable short-term follow-up information concerning pain relief was available in 1659 radiotherapy series. In $79.3 \%$ of the cases patients were painless or experienced marked or at least slight pain relief immediately or up to two months after the completion of radiotherapy (Figure 1). Neither gender (Figure 2) nor age (cutoff 60 years) (Figure 3) had a significant influence on the painrelieving effect. Figure 4 refers to 471 joints with complete information on the radiological severity of osteoarthritis prior to irradiation. In $84.9 \%$ of the cases with severe signs of osteoarthritis radiotherapy led to slight or marked pain-relief. In the cases with minimal / moderate signs of osteoarthritis this result was only achieved in $77.2 \% / 78.3 \%$. The difference proved to be statistically significant $(\mathrm{p}<0.05)$. There was no significant difference in radiation-induced pain relief between the patients who reported a history of pain of less than a year, 1 to 3 years or more than 3 years prior to radiotherapy (Figure 5).

\section{Response to treatment as recorded by the additional mail survey}

It was found that a total of $49.1 \%$ (39.8\%) of the respondents had indicated a beginning or significant pain relief

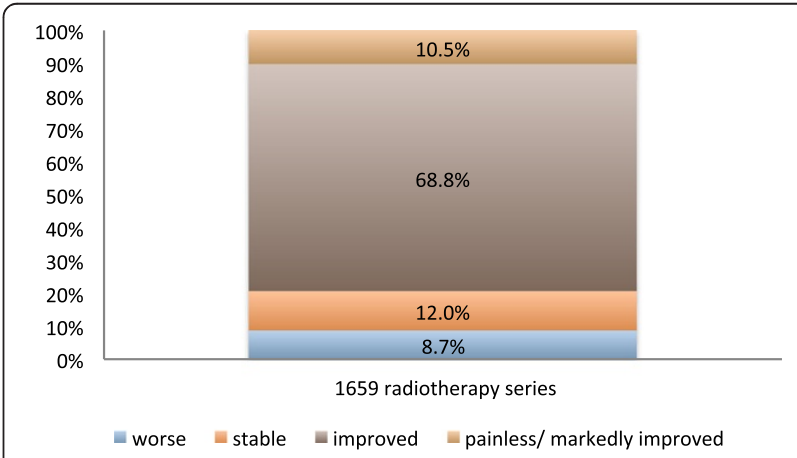

Figure 1 Overall response to radiotherapy. Pain, as it was subjectively graded by the patients immediately or up to two months after the completion of a series of radiotherapy. 


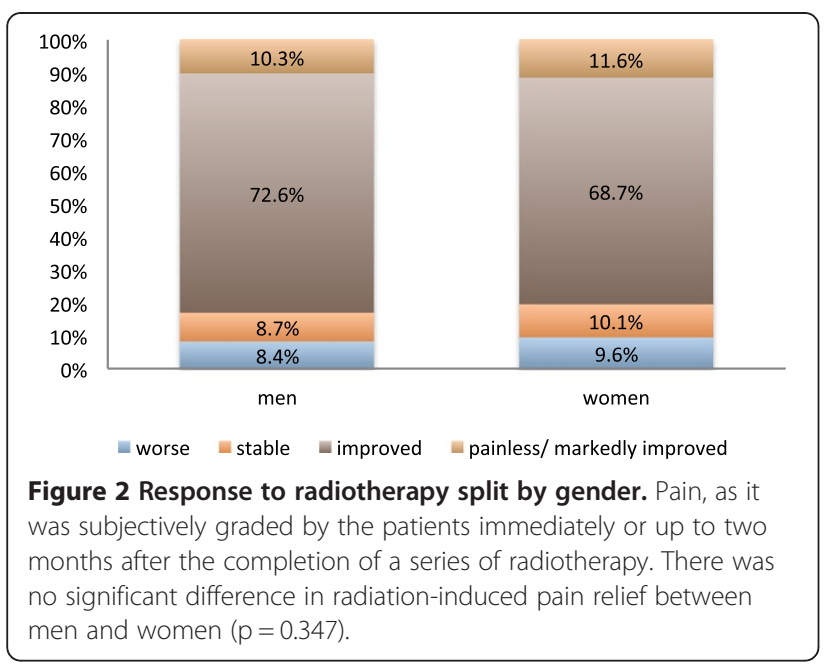

(mobility improvement) after treatment (Figure 6 \& Figure 7). In more than $50 \%$ of the patients who reported a positive response to irradiation a sustained period of symptomatic improvement was observed (Figure 8).

\section{Discussion}

\section{Best response to radiotherapy}

Similar to the previous studies identified by our literature search this large retrospective analysis demonstrates the good analgetic effect of radiotherapy in painful osteoarthritis of the knee. In $80 \%$ of the cases the irradiated patients reported a positive response to treatment immediately or up to two months after the completion of the respective series. However, the proportion of very good responders, i.e., patients who were free of pain or in whom the symptoms had markedly improved after a series of radiotherapy, was low in comparison to the other series (Additional file 1: Table S1). A possible explanation for that phenomenon could be that, for practical reasons, the response to treatment had been evaluated more likely

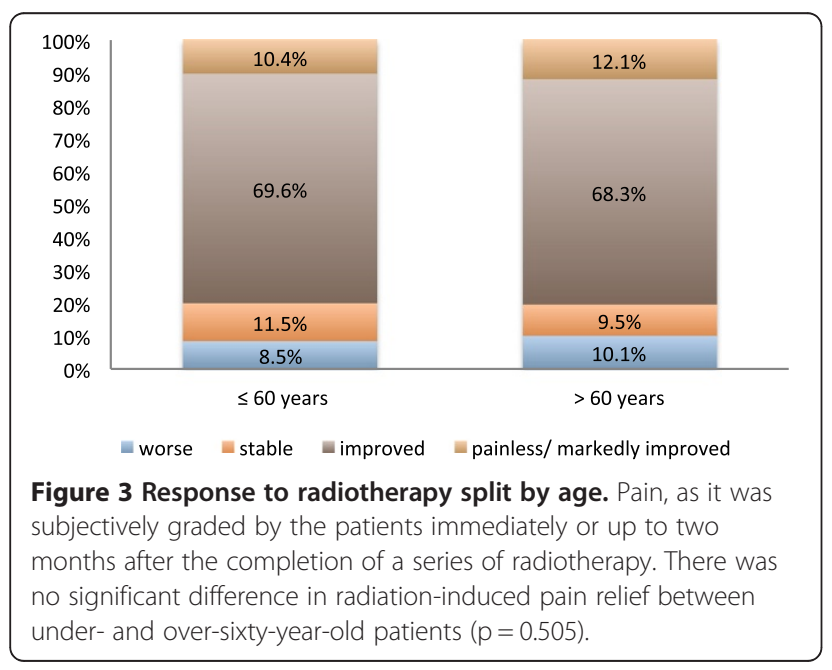

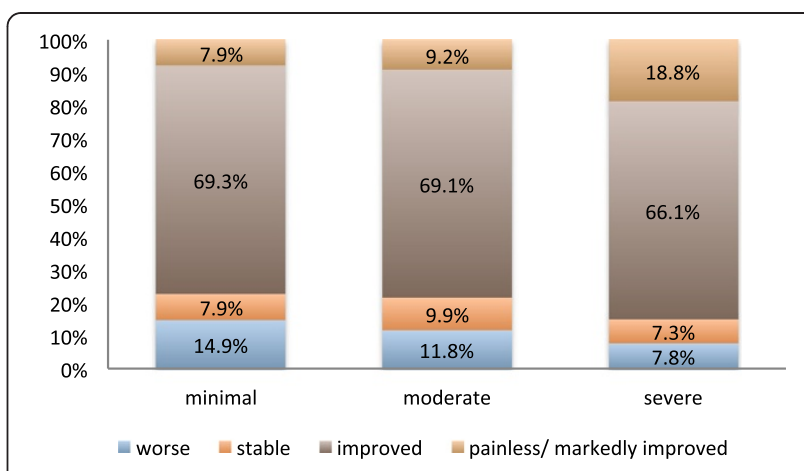

Figure 4 Response to radiotherapy split by radiological severity of gonarthritis. Pain, as it was subjectively graded by the patients immediately or up to two months after the completion of a series of radiotherapy. There was a significant difference in radiation-induced pain relief between minimal/ moderate and severe gonarthritis $(p=0.036)$.

immediately rather than several weeks after the completion of radiotherapy.

\section{Point of time of the best response to radiotherapy}

The assessment of the treatment effect should be performed immediately and a few weeks after the completion of radiotherapy, since in a high percentage of cases outcome apparently improves over time. Several analyses demonstrate that approximately ten percent of the patients are free of symptoms or feel significant pain relief directly after the completion of radiotherapy $[10,13]$. In the cohort assessed by Keinert et al. the proportion of patients with complete resolution of symptoms increased from 8 to 38 percent in the following six weeks [10]. In a cohort of 21 patients assessed by Sautter-Bihl et al. the final treatment success occurred in 10\% during, in $14 \%$ immediately after and in $76 \%$ within six weeks after

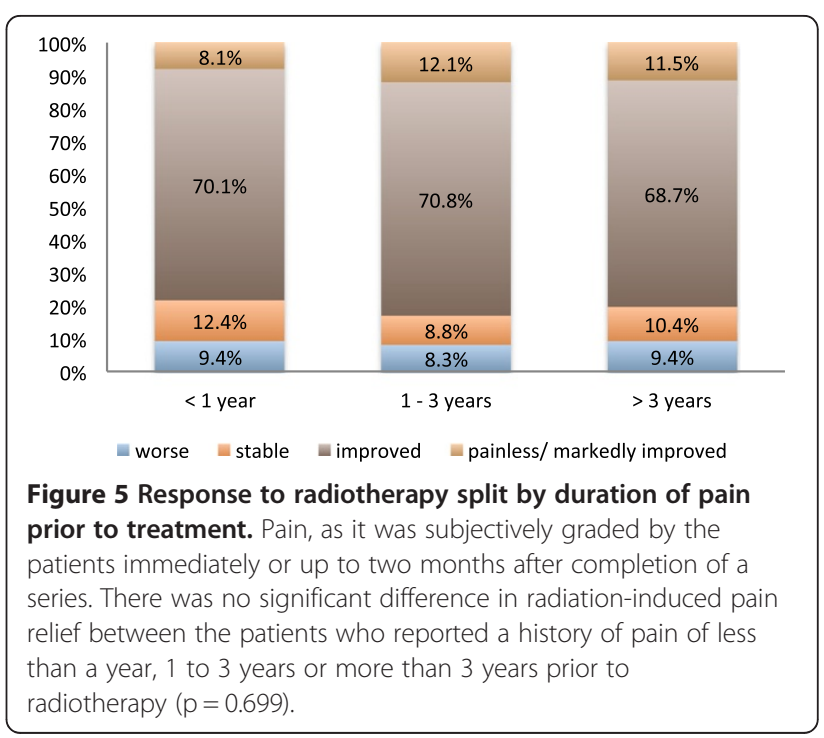




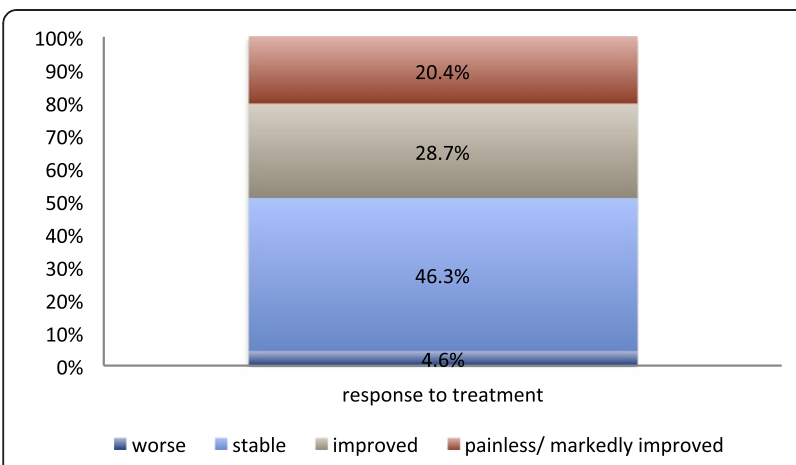

Figure 6 Response to radiotherapy according to the additional mail survey (106 evaluable questionnaires). Pain after the end of radiotherapy, as it was subjectively graded by the patients in a retrospective mail survey, which was effected in 2010, i.e., two to fourteen years after treatment.

radiotherapy [13]. Accordingly Ruppert et al. investigated a cohort of 73 patients with painful osteoarthritis affecting different joints and reported a delay of ten weeks between the start of radiotherapy and by pain reduction in $47 \%$ of the patients who responded to treatment [12].

\section{Duration of the analgetic effect}

$57 \%$ of the responders in our cohort experienced notable pain-relief for more than a year (Figure 8). In contrast, Mücke et al. reported a median estimate for the share of patients who experienced pain reduction for at least 12 months of only $40 \%$ [5]. In the cohort of patients assessed by Sautter-Bihl et al. the pain-relieving effect of radiotherapy lasted longer than 12 months in $67 \%$ of the cases [13]. In the analysis performed by Keinert et al. the share of the responders who suffered a recurrence ranged between 30\% (patients with marked pain-relief) and 50\% (patients who were free of pain after radiotherapy) [10].

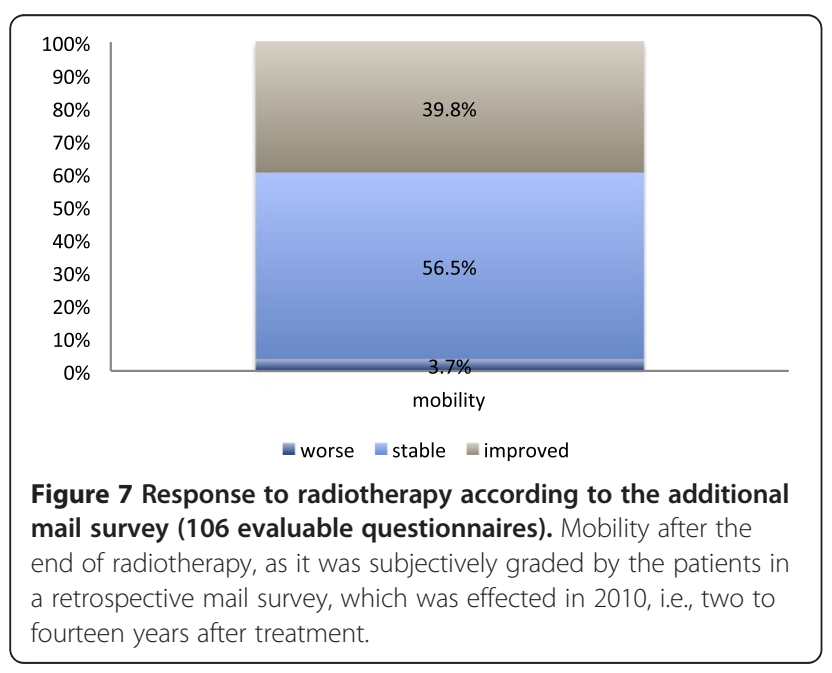

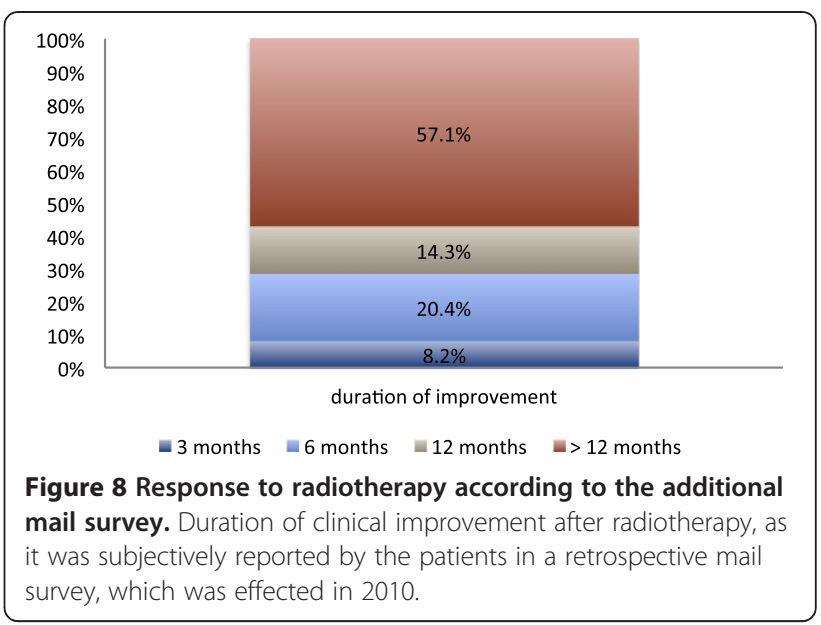

This observation was confirmed by Keilholz at al. who found a relapse rate of $33 \%$ in a cohort of 30 responders [11]. The analgetic potential of radiotherapy in painful osteoarthritis of the knee seems to be comparable to that in other big joints [12].

\section{Prognostic factors \\ Gender and age}

Neither gender (Figure 2) nor age (Figure 3) had a significant influence on the pain-relieving effect in this retrospective study. In contrast in the analysis performed by Keilholz et al. the patients $<80$ years tended to have a more favorable treatment response (univariate analysis, $\mathrm{p}=0.08)$ [11]. However this trend could not be confirmed in multivariate analysis. In the study of Glatzel et al. age ( $\leq 60$ years versus $>60$ years) did not influence outcome whereas best results with a clear analgesic effect were reached in males (29/50, 58\% versus femals: 39/135, 29\%). The factor gender had an independent prognostic value $(\mathrm{p}<0.01)$ [14].

\section{Severity}

The impact of the radiological severity of osteoarthritis on the response to radiotherapy remains controversial. In this study in $84.9 \%$ of the cases with severe signs of osteoarthritis the first irradiation series led to slight or marked pain-relief. In the cases with minimal/ moderate signs of osteoarthritis this result was only achieved in $77.2 \% / 78.3 \%$. The difference proved to be significant. In contrast, in the analysis released by Keilholz et al. the patients with severe radiological signs of osteoarthritis tended to respond worse to radiotherapy in univariate analysis [11].

\section{Duration of symptoms}

Some authors stated that the results of treatment were dependent on the duration of symptoms before the start of radiotherapy. In the cohort of Keinert et al. $48 \%$ of 
the patients with a short duration of symptoms ( $<1$ year) were pain-free after irradiation. In patients with a longer duration of symptoms ( $>1$ year) this result was only achieved in $25 \%$. Keinert et al. derived from their observations the demand to use irradiation, contrary to the usual practice, as early treatment option [10]. This demand was supported by Keilholz et al. and Glatzel et al. who demonstrated that a short duration of pain symptoms before the start of radiotherapy $(\leq 2$ years and $\leq 1$ year respectively) was an independent positive prognostic factor for the success of pain-relieving radiotherapeutic treatment in multivariate analysis $(\mathrm{p}<0.05)$ $[11,14]$. In contrast, in our cohort there was no significant difference in radiation-induced pain relief between the patients who reported a history of pain of less than a year, 1 to 3 years or more than 3 years prior to radiotherapy.

\section{$R T$-technique and dosage}

The optimal radiotherapy technique and dosage are currently unknown. Frequently two opposed irradiation fields are used and the reference point of the dose is placed in the center of the joint. However, assuming a typical knee diameter of $10 \mathrm{~cm}$, the clinical relevance of this approach may be questioned, as the respective dose distributions within the knee joint will diverge only marginally if the reference point is shifted to the skin surface (Additional file 1: Figure S1). Although in our cohort the use of alternating single fields was associated with a better response to treatment (data not shown), we are reluctant to derive radiotherapy planning recommendations from those findings as our results may be biased by the variety of additional factors influencing the dose distribution within the knee joint (i.g. acceleration voltage, thickness of the filter, focus-skin distance, field size, diameter of the knee) by different dose prescriptions and fractionation schemes (Table 1) or other not treatment related factors.

According to Mücke et al. most institutions in Germany irradiate with a median single dose of 1 Gy twice (40\%) or three times (51\%) a week and a median total dose of 6 Gy [5].

In our institutions the majority of patients received single doses of 1 Gy (83.7\%). A total doses of 6 Gy was only administered in $7.4 \%$ of the cases. Most patients (54.2\%) received a total dose of 4 Gy. About two thirds of our patients underwent irradiation once and one third twice a week. A limitation of our analysis is that the influence of different dose-fractionation schedules on treatment response in painful gonarthritis could not be assessed. Niewald et al. conducted a randomized trial of radiation therapy for painful heel spur, comparing a standard dose with a very low dose. Sixty-six patients were randomized to receive radiation therapy either with a total dose of 6.0 Gy applied in 6 fractions of 1.0 Gy twice weekly (standard dose) or with a total dose of
0.6 Gy applied in 6 fractions of 0.1 Gy twice weekly (low dose). After 3 months the results in the standard arm were significantly superior compared with those in the low-dose arm. The accrual of patients was stopped at this point [15]. Heyd et al. evaluated the efficacy of two different dose-fractionation schedules for radiation therapy (RT) in patients with painful heel spur. 130 patients were randomized into two groups: the low-dose (LD) group ( $\mathrm{n}=65$ heels) received a total dose of 3.0 Gy given in two weekly fractions of $0.5 \mathrm{~Gy}$; in the high-dose (HD) group ( $\mathrm{n}=65$ heels), two weekly fractions of 1.0 Gy were applied over 3 weeks (total dose 6.0 Gy). No statistically significant difference of response to RT between both groups was observed [16]. These results are in accordance with the findings of Ott et al. who evaluated the efficacy of two different dose-fractionation schedules for radiotherapy of patients with painful elbow syndrome. One RT course consisted of 6 single fractions/ 3 weeks. Patients were randomly assigned to receive either single doses of 0.5 or $1.0 \mathrm{~Gy}$. Endpoint was pain reduction. No statistically significant differences between the two single dose trial arms for early $(\mathrm{p}=0.103)$ and delayed response $(\mathrm{p}=0.246)$ were found [17]. Liebmann et al. explored the efficacy of low-dose radiotherapy in adjuvant induced gonarthritis in rats using different fractionation schemes to specify a possible dose and fractionation dependence. Based on the experimental data they recommended two series of $5 \times 0.5$ Gy with an early treatment onset and repetition in interval during the florid phase of arthritis as most effective radiotherapy regimen to prevent a full-blown arthritic reaction [18].

\section{Mechanism of action}

Pathophysiology of osteoarthritisis has not yet been understood completely. However, arthrosis, i.e. the degeneration of articular cartilage, leads to an inflammatory reaction in the synovial membrane which again aggravates arthrosis [19]. Several authors showed in animal models that low-dose radiotherapy attenuates the arthritic response by anti-inflammatory effects and decreases its clinical symptoms $[9,18,20]$.

\section{Risk of somatic damage and malignant transformation}

The risks of radiation exposure always have to be weighed against the therapeutic benefit. Somatic damage is not expected at the given low doses [10]. Moreover since most patients are seniors damage to the genetic material plays only a minor role. According to Jansen et al. the average attributable lifetime risk for an induced fatal tumor for a $25 / 50 / 75$-year-old woman is $4 / 1 / 0.5 \%$ o for a double series treatment with a target dose of 12 Gy. For a single series these values are 2.0, 0.7 and 0.3 respectively [21]. Despite these considerations 
compliance with all health and safety rules remains a matter of course for each radiotherapist.

\section{Conclusions}

In accordance with previous retrospective analyses, especially the large pattern of care study of the German Cooperative Group on Radiotherapy for Benign Diseases, our results confirm that low-dose radiotherapy is an effective treatment for painful osteoarthritis of the knee. The influence of radiological severity on treatment outcome remains unclear. In contrast to an earlier retrospective study we identified severe signs of osteoarthritis as positive prognostic factor for treatment response. A randomized trial is urgently required to compare radiotherapy with other therapy methods.

\section{Additional file}

Additional file 1: Table S1. Outcome of radiotherapy in painful gonarthritis. Overview of literature results in radiotherapy for painful gonarthritis (1980-2012) including our study, modified classification of therapy response according to von Pannewitz [22].* very good response = painless $/$ markly improved, good/ satisfying response = improved, little/ no response $=$ stable, ${ }^{* *}$ identical patient cohort . Figure S1: Dose distribution in a knee (coronar view). Dose distribution in a knee with a diameter of $10 \mathrm{~cm}$ as a function of different irradiation techniques using an orthovoltage unit with $175 \mathrm{kV}, 20 \mathrm{~mA}, 0.5 \mathrm{~mm}$ copper filter, focus-skin distance $40 \mathrm{~cm}$ and lateral (opposed) fields $(10 \mathrm{~cm} \times 15 \mathrm{~cm}$ ).

\section{Competing interests}

The authors declare that they have no competing interests.

\section{Authors' contributions}

SK was responsible for the collection of data, statistical evaluation and writing of the manuscript. KM was responsible for the check of the data, statistical evaluation, review of the literature and writing of the manuscript. RDK, GH and DB were responsible for the conception of the study. DB and GF were responsible for the treatment of the majority of patients and the control of the documentation of the treatment and follow-up data. AL, UW and OM critically evaluated and approved the manuscript. All authors read and approved the final manuscript.

\section{Acknowledgements}

The authors wish to acknowledge Ms. Christiane Hofmann for her meticulous literature search.

\section{Author details}

${ }^{1}$ Department of Internal Medicine II- Gastroenterology / Hepatology / Oncology / Infectology / Tropical Medicine /Endocrinology / Diabetology, Klinikum Chemnitz gGmbH, Flemmingstraße 2, Chemnitz 09116, Germany. ${ }^{2}$ Department of Radiotherapy, University Hospital of Leipzig, Stephan-Str. 9a, Leipzig 04103, Germany. ${ }^{3}$ Department of Radiotherapy, University Medicine Rostock, Südring 75, Rostock 18059, Germany. ${ }^{4}$ Department of Radiotherapy, Franziskus Hospital Bielefeld, Kiskerstraße 26, Bielefeld 33615, Germany. ${ }^{5}$ Department of Radiology, Heliosklinikum Aue, Gartenstraße 6, 08280, Aue, Germany. ${ }^{6}$ Department of Radiotherapy, Klinikum Chemnitz GmbH, Flemmingstraße 2, Chemnitz 09116, Germany.

Received: 22 November 2012 Accepted: 20 January 2013

Published: 31 January 2013

\section{References}

1. Felson DT: Osteoarthritis. Rheum Dis Clin North Am 1990, 16:499-512.

2. Engelhardt M: Epidemiologie der arthrose in westeuropa. Dtsch Z Sportmed 2003, 54:171-175.
3. Fried G: Die röntgentherapie der arthritis. Strahlentherapie 1934, 49:634-675.

4. Toschke G: Zur röntgenbehandlung von gelenkerkrankungen. Strahlentherapie 1941, 70:443-456.

5. Mücke R, Seegenschmiedt MH, Heyd R, Schafer U, Prott FJ, Glatzel M, Micke O: Radiotherapy in painful gonarthrosis. Results of a national patterns-of-care study. Strahlenther Onkol 2010, 186:7-17.

6. Jordan KM, Arden NK, Doherty M, Bannwarth B, Bijlsma JW, Dieppe P, Gunther K, Hauselmann H, Herrero-Beaumont G, Kaklamanis $P$, et al: EULAR recommendations 2003: an evidence based approach to the management of knee osteoarthritis: report of a task force of the standing committee for international clinical studies including therapeutic trials (ESCISIT). Ann Rheum Dis 2003, 62:1145-1155.

7. Kellgren JH, Lawrence JS: Osteo-arthrosis and disk degeneration in an urban population. Ann Rheum Dis 1958, 17:388-397.

8. Von Pannewitz G (Ed): Roentgen therapy for deforming arthritis. Leipzig: Thieme; 1933.

9. Fischer U, Kamprad F, Koch F, Ludewig E, Melzer R, Hildebrandt G: The effects of low-dose Co-60 irradiation on the course of aseptic arthritis in a rabbit knee joint. Strahlenther Onkol 1998, 174:633-639.

10. Keinert KSU, Schumann E: Ergebnisse der strahlentherapie der arthrosis deformans des kniegelenks. Dt Gesundh-Wesen 1982, 10:445-447.

11. Keilholz L, Seegenschmiedt H, Sauer R: Radiotherapy for painful degenerative joint disorders. Indications, technique and clinical results. Strahlenther Onkol 1998, 174:243-250.

12. Ruppert R, Seegenschmiedt MH, Sauer R: Radiotherapy of osteoarthritis Indication, technique and clinical results. Orthopade 2004, 33:56-62.

13. Sautter-Bihl ML, Liebermeister $E$, Scheurig H, Heinze HG: Analgetic irradiation of degenerative-inflammatory skeletal diseases. Benefits and risks. Dtsch Med Wochenschr 1993, 118:493-498.

14. Glatzel MF D, Bäsecke S, Krauß A: Prognostic factors of success of analgesic radiotherapy for gonarthrosis. Strahlenther Onkol 2004, 180(Sondernr 1):6.

15. Niewald M, Seegenschmiedt MH, Micke O, Graeber S, Muecke R, Schaefer $V$, Scheid C, Fleckenstein J, Licht N, Ruebe C: Randomized, multicenter trial on the effect of radiation therapy on plantar fasciitis (painful heel spur) comparing a standard dose with a very low dose: mature results after 12 months' follow-up. Int J Radiat Oncol Biol Phys 2012, 84:e455-462.

16. Heyd R, Tselis N, Ackermann H, Roddiger SJ, Zamboglou N: Radiation therapy for painful heel spurs: results of a prospective randomized study. Strahlenther Onkol 2007, 183:3-9.

17. Ott OJ, Hertel S, Gaipl US, Frey B, Schmidt M, Fietkau R: Benign painful elbow syndrome: first results of a single center prospective randomized radiotherapy dose optimization trial. Strahlenther Onkol 2012, 188:873-877.

18. Liebmann A, Hindemith M, Jahns J, Madaj-Sterba P, Weisheit S, Kamprad F Hildebrandt G: Low-dose X-irradiation of adjuvant-induced arthritis in rats. Efficacy of different fractionation schedules. Strahlenther Onkol 2004, 180:165-172.

19. Trott KR, Parker R, Seed MP: The effect of x-rays on experimental arthritis in the rat. Strahlenther Onkol 1995, 171:534-538.

20. Hildebrandt G, Jahns J, Hindemith M, Spranger S, Sack U, Kinne RW, Madaj-Sterba $P$, Wolf $U$, Kamprad F: Effects of low dose radiation therapy on adjuvant induced arthritis in rats. Int J Radiat Biol 2000, 76:1143-1153.

21. Jansen JT, Broerse JJ, Zoetelief J, Klein C, Seegenschmiedt HM: Estimation of the carcinogenic risk of radiotherapy of benign diseases from shoulder to heel. Radiother Oncol 2005, 76:270-277.

22. Von Pannewitz G: Röntgentherapie der arthrosis deformans. Strahlentherapie 1953, 92:375-382

doi:10.1186/1748-717X-8-29

Cite this article as: Keller et al: Efficacy of low-dose radiotherapy in painful gonarthritis: experiences from a retrospective East German bicenter study. Radiation Oncology 2013 8:29. 